\title{
Evaluation nutzen!
}

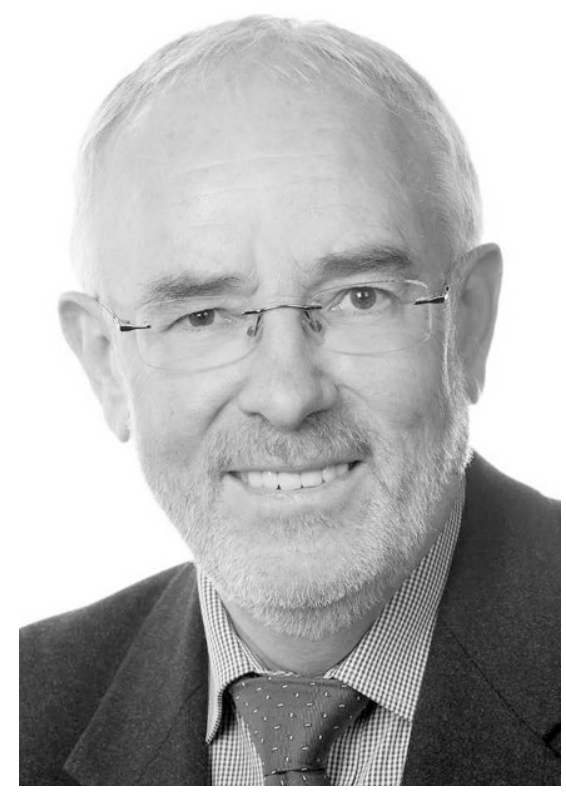

Art. 97 Abs. 1 DSGVO fordert von der Europäischen Kommission, vier Jahre nach Inkrafttreten und zwei Jahre nach Geltungsbeginn, bis zum 25. Mai 2020 einen Bericht über die Bewertung und Überprüfung der Datenschutz-Grundverordnung vorzulegen. Von da an soll eine solche Evaluation der Verordnung alle vier Jahre wiederholt werden.

Die Regelung des Art. 97 DSGVO zur regelmäßigen Evaluation der Verordnung gibt der Erkenntnis Ausdruck, dass die Digitalisierung Wirtschaft, Staat und Gesellschaft sehr schnell und sehr nachhaltig verändert und dass der Schutz der Werte, die in diesem Wandel unverändert bleiben sollen, sich immer wieder neuen Herausforderungen anpassen muss. Art. 97 DSGVO ist auch Ausdruck des Wissens um den Entwurfscharakter der Datenschutz-Grundverordnung. Sie ist eine erste Fassung einer unionsweiten Datenschutzregelung, deren Autoren die vielen und vielfältigen Praxisprobleme in allen von ihr erfassten Wirtschafts-, Verwaltungs- und Gesellschaftsbereichen gar nicht kennen konnten. Sie ist außerdem eine Sammlung von unterschiedlichen, nur mühsam systematisierten Kompromissergebnissen, die bei den vieldimensionalen Interessengegensätzen und den 2015 gegebenen Machtverhältnissen durchsetzbar waren. Sie ist somit ein legislativer Versuch, der angesichts neuer Herausforderungen für Persönlichkeitsrechte und Demokratie immer wieder neu zu konzipieren und zu verhandeln ist.

Aufgrund ihres Anspruchs, Datenschutz als Querschnittsaufgabe für die Digitalisierung aller Gesellschaftsbereiche für die gesamte Europäische Union mit vergleichsweise wenigen Vorgaben einheitlich zu regeln, muss sie hochabstrakt sein und kann viele Regelungen nur andeuten. Dies hinterlässt in der Praxis notwendigerweise vielfältige Auslegungsspielräume. Diese ermöglichen Anpassungen der Vorgaben an unterschiedliche Praxisherausforderungen. Sie werden aber zu Defiziten der Datenschutzpraxis, wenn sie Rechtsunsicherheit, Investitionsstau, Vollzughemmnisse, Unverständnis und Handlungsbarrieren verursachen. Vielfach versuchen Berater, Anwälte, Literatur und Aufsichtsbehörden, nach und nach auch der Europäische Datenschutzsauschuss, diese Defizite durch entsprechende Auslegungsversuche zu beseitigen. Diese führen jedoch immer zu interessengeleiteten Meinungsstreitigkeiten, die erst nach und nach mit hohem Aufwand und großem Zeitverlust aufgelöst werden können. Außerdem zeigt die Erfahrung, dass überall da, wo das Recht normative Spielräume eröffnet, letztlich soziale, politische und wirtschaftliche Macht eindringt und einseitige Ergebnisse durchsetzt.

Bis letztlich der Europäische Gerichtshof in Einzelfällen die Defizite beseitigt und für Rechtssicherheit und Interessenausgleich sorgt, vergeht geraume Zeit. Vielfach hat die Dynamik der technischen Entwicklung das Problem dann bereits überholt. Da der Gerichtshof an den Text der Datenschutz-Grundverordnung gebunden ist, dürfte ihm in vielen Fällen die gebotene Korrektur auch gar nicht möglich sein. Schließlich sorgt nicht jede Entscheidung des Gerichts für Klarheit, sondern hinterlässt - wie bei der gemeinsamen Verantwortung - mehr Fragen als vorher bestanden. Daher sollte der europäische Gesetzgeber diese Defizite, die er ja verursacht hat, möglichst bald beheben. Die jeweiligen Verbesserungsmöglichkeiten sollten daher das zentrale Thema in der Evaluation durch die Europäische Kommission sein.

Art. 97 DSGVO gibt einen Weg vor, wie die Umsetzung der Datenschutz-Grundverordnung in der Praxis kontinuierlich überprüft und sowohl die kleinen Verbesserungen im Verordnungstext als auch eine umfassende Modernisierung der Datenschutzkonzeption im Rahmen einer Evolution des Datenschutzrechts ermöglicht werden können.

Um dazu beizutragen, diese Chance zur Verbesserung der Datenschutz-Grundverordnung zu nutzen, lohnt es, über das Verfahren der Evaluation zu informieren, Erfahrungen mit der Datenschutz-Grundverordnung aus unterschiedlichen Perspektiven zu sammeln, auf Defizite der Verordnung hinzuweisen, über vorliegende Be- 


\section{算 Springer Vieweg}

R. Schuhmann, B. Eichhorn (Hrsg.) Contractual Management

Managing Through Contracts

2020, XXXIII, 404 p. 38 illus., 23 illus. in color. Geb.

$€$ (D) $53,49 \mid €\left(\right.$ A) $54,99 \mid{ }^{*}$ CHF 66.61

ISBN 978-3-662-58481-1

$€ 42,79 \mid{ }^{*}$ CHF 53.00

ISBN 978-3-662-58482-8 (eBook)

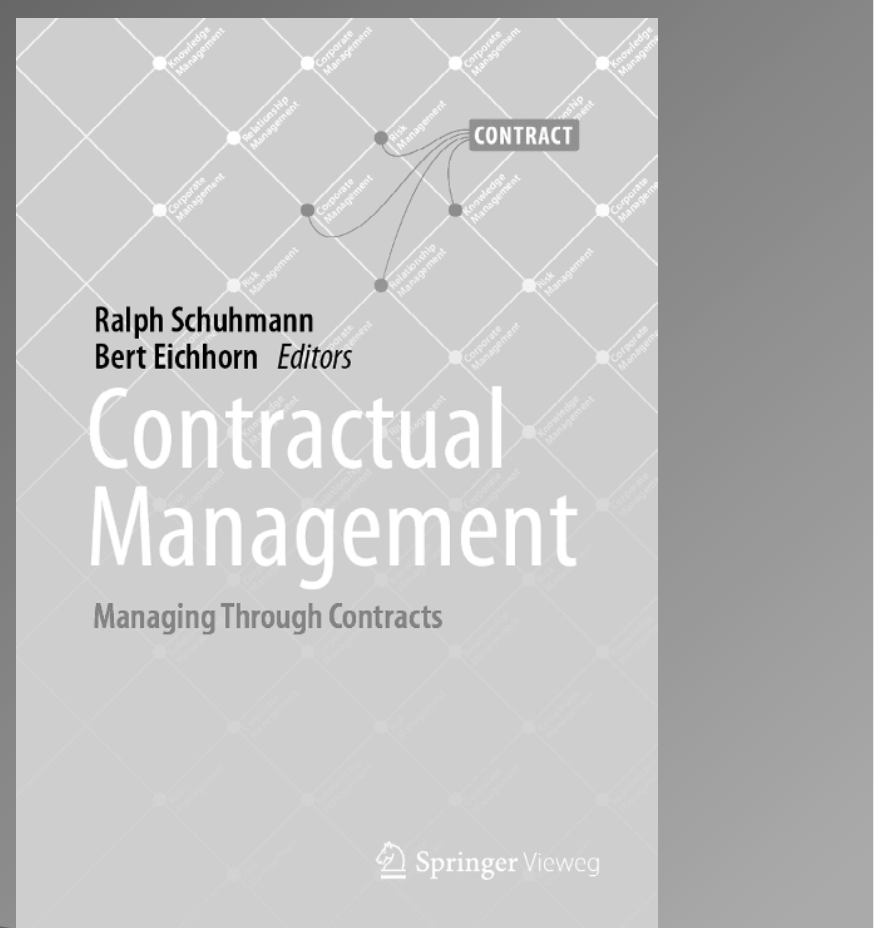

\section{Vertragsmanagement neu gedacht}

- Explores the theoretical foundations of contractual management

- Covers a variety of industries, company sizes, contract types, and management situations

- Case studies illustrate applications of contract management

The Concept Contractual Management offers a holistic approach to managerial decision-making based on contracts or business processes that are related to contracts. It explains management from the point of view of the contract, just as it interprets the contract from the point of view of management. Thus, the approach highlights the great inherent potential of contracts for managing companies, transactions and business relationships. The book addresses students as well as practitioners and gives insights into the usage of contracts to manage companies or relationships.

Ihre Vorteile in unserem Online Shop:

Über 280.000 Titel aus allen Fachgebieten | eBooks sind auf allen Endgeräten nutzbar | Kostenloser Versand für Printbücher weltweit

$€(D)$ : gebundener Ladenpreis in Deutschland, $€(A)$ : in Österreich. * : unverbindliche Preisempfehlung. Alle Preise inkl. MwSt. 
wertungen und Verbesserungsvorschläge zu berichten und eigene Vorschläge zu entwerfen.

Zu dieser Diskussion wollen die Beiträge im Schwerpunkt dieses Heftes beitragen. Sie sind zur Vorbereitung der Veranstaltung „Evaluation der Datenschutz-Grundverordnung - Welche Verbesserungen sind notwendig?" entstanden, die das Competence Center for Applied Security Technology (CAST) und das BMBF-Forum „Privatheit und selbstbestimmtes Leben in einer digitalen Welt" gemeinsam am 12. März 2020 in Darmstadt veranstalten wollten. Sie konnte wegen der Corona-Krise leider nicht stattfinden.

Der einleitende Beitrag von Roßnagel, Universität Kassel, erläutert das Verfahren und die Vorgaben des Art. 97 DSGVO zur Evaluation der Datenschutz-Grundverordnung und gibt einen Überblick über die bisherige Diskussion. Hierzu stellt er wichtige bis zum 1. März 2020 veröffentlichte Stellungnahmen zur Evaluation vor und bewertet in systematisierender Weise die in ihnen enthaltenen Vorschläge zur Verbesserung der Verordnung. Der folgende Beitrag von Weichert vom Netzwerk Datenschutzexpertise eröffnet einen Diskussionsraum für die Evaluation der DatenschutzGrundverordnung, indem er diese in den Zusammenhang eines globalen digitalen Grundrechtsschutzes stellt. Für diesen kann die Verordnung als Vorbild dienen, gerade wenn sie noch viele Schutzlücken schließt und Inkonsistenzen beseitigt. Jaspers und Jaquemain von der Gesellschaft für Datenschutz und Datensicherheit berichten über Erfahrungen mit der Umsetzung der Datenschutz-Grundverordnung in Vereinen und in kleinen und mittleren Unternehmen und schlagen Verbesserungen vor allem aus der Perspektive von Datenschutzbeauftragten vor. Schulz, Rechtsanwalt in Berlin, zieht Schlussfolgerungen aus seiner Beratungspraxis und entwickelt aus seinen Erfahrungen in der praktischen Umsetzung der Datenschutz-Grundverordnung Vorschläge, sie praxisgerechter zu gestalten. Hierbei geht es ihm vor allem um die Beschränkung von Kommunikationspflichten (Informationen, Auskunft und Kopie) und maßvolle Begrenzungen von Betroffenenrechten. Die beiden letzten Beiträge befassen sich mit zwei inhaltlichen Themen, die in den bisherigen Stellungnahmen zur Evaluierung eher unterbelichtet sind: Geminn, Universität Kassel, untersucht, welche Defizite die Verordnung hinsichtlich der Rechte der betroffenen Person aufweist und präsentiert viele Verbesserungsvorschläge für den Text der Verordnung, diese zu beseitigen und den Grundrechtsschutz zu stärken. Glatzner, Verbraucherzentrale Bundesverband, analysiert die Lücken und Fehlanreize in der Regulierung algorithmenbasierter Entscheidungssysteme. Zu ihrer Beseitigung schlägt er Klarstellungen und Verbesserungen der Datenschutz-Grundverordnung vor, aber auch Regelungen, die über den rechtlichen Rahmen des Datenschutzes hinausgehen.

Alle Beiträge kommen - auf unterschiedlichen Wegen und mit unterschiedlicher Gewichtung - letztlich zu dem Ergebnis, dass es der Datenschutz-Grundverordnung an der notwendigen Rechtssicherheit mangelt und dass es sich lohnt, über Verbesserungen ihrer Regelungen zu diskutieren und sich für ihre Verwirklichung einzusetzen.

\section{Alexander Roßnagel}

